\title{
NSAIDs erhöhen Risiko für Bluthochdruck bei AS-Patienten
}

\author{
Liew JW et al. Nonsteroidal Antiinflammatory \\ Drug Use and Association With Incident \\ Hypertension in Ankylosing Spondylitis. Arthritis \\ Care Res 2020; 72: 1645-1652
}

Patienten mit ankylosierender Spondylitis (AS) haben ein erhöhtes Risiko für kardiovaskuläre Erkrankungen. Aktuelle Leitlinien empfehlen nichtsteroidale Antirheumatika (NSAIDs) als First-Line Therapie der AS, Studien zeigen jedoch, dass NSAIDs den Blutdruck erhöhen können. Liew et al untersuchten die Assoziation einer NSAID-Therapie mit der Entwicklung einer Hypertonie in einer großen prospektiven Beobachtungskohorte von AS-Patienten.

Die Resultate dieser prospektiven, longitudinalen Beobachtungsstudie zeigen, dass eine kontinuierliche Einnahme von NSAIDs im Vergleich zu nicht kontinuierlicher oder keiner NSAID-Einnahme mit einem um 12\% erhöhten Risiko für die Entwicklung von Bluthochdruck einhergeht. Die Forscher rekrutierten die Studienprobanden mit einem Alter von $\geq 18$ Jahren aus insgesamt 5 Zentren in den USA und Australien. Bei Studienbeginn erfassten die Wissenschaftler die demografischen Daten der Patienten, die Charakteristika des AS-Krankheitsstatus, den HLA-B27-Status, das Datums des Symptombeginns, extraartikuläre Manifestationen, eventuelle Komorbiditäten und die Medikamentenanamnese. Alle 2 Jahre führten die Ärzte eine Follow-Up-Visite durch, im Rahmen derer die Patienten auf Komorbiditäten wie Hypertonien, koronare Herzkrankheiten, Herzklappenerkrankungen, eine Vorgeschichte von Herzinfarkt, koronare Revaskularisation, koronare BypassOperationen, Diabetes mellitus, Nierenerkrankungen und psychische Erkrankungen untersucht wurden. Im Abstand von 4-6 Monaten beurteilten die Ärzte die Krankheitsaktivität und die funktionellen Beeinträchtigungen der Patienten anhand des Bath Ankylosing Spondylitis Disease Activity Index (BASDAI) und des Ankylosing Spondylitis Disease Activity Score (ASDAS). Für alle Medikamente, die die Patienten in den vorangegangenen 6 Monaten einge- 
nommen hatten, dokumentierten die Experten im Patientenbericht die Dosierung, Häufigkeit und Dauer. Zusätzlich bestimmten die Forscher die jeweiligen Spiegel des C-reaktiven Proteins (CRP), die Erythrozytensedimentationsrate und Vitalparameter einschließlich Blutdruck und Body-Mass-Index (BMI) bei jeder Visite. Den NSAID-Verbrauch quantifizierten die Forscher anhand des NSAID-Indexes gemäß den Empfehlungen der internationalen Gesellschaft zur Beurteilung von Spondyloarthritis.

Die Forscher schlossen 628 Personen mit ankylosierender Spondylitis in die Studie ein. Das Durchschnittsalter bei Studieneintritt betrug $39 \pm 13$ Jahre, $72 \%$ der Patienten waren männlich, $80 \%$ waren weiß. Insgesamt $43 \%$ nahmen Biologika, insbesondere Tumor-Nekrose-Faktor-Inhibitoren (TNFi) und $7 \%$ nahmen Glukokortikoide ein. $2 \%$ der Patienten litten an kardiovaskulären Erkrankungen, $1 \%$ hatte Diabetes mellitus und $3 \%$ gaben an, ein Statin einzunehmen. 200 Patienten berichteten über eine kontinuierliche NSAID-Einnahme und 428 über eine entweder niedrig dosierte oder keine NSAID-Einnahme. Die demografischen Merkmale zwischen den beiden Gruppen waren ähnlich, jene Patienten, die zu Beginn der Studie kontinuierlich NSAR einnahmen, wiesen jedoch eine signifikant höhere Krankheitsaktivität nach BASDAI als auch nach ASDAS auf, zudem zeigte ein größerer Anteil erhöhte CRP-Werte. Während einer medianen Nachbeobachtungszeit von 7,0 Jahren diagnostizierten die Ärzte bei 129 Patienten einen neu aufgetretenen Bluthochdruck. Von diesen Patienten nahmen zu Studienbeginn $40 \%$ kontinuierlich NSAR ein, $47 \%$ TNFi und $16 \%$ nahmen sowohl regelmäßig NSAR als auch TNFi ein. Nach einer Anpassung des statistischen Modells an Studienort, Alter, Geschlecht, Rasse, BMI, TNFi-Einnahme und Krankheitsaktivität nach ASDAS erwies sich eine kontinuierliche NSAID-Einnahme als mit einem erhöhten Risiko für das Auftreten von Bluthochdruck assoziiert. Dieser Effekt war bei Patienten mit unregelmäßiger oder keiner NSAID-Einnahme nicht der Fall.

\section{FAZIT}

Die Ergebnisse dieser Studie zeigen, dass eine kontinuierliche Einnahme nichtsteroidaler Antirheumatika mit einem erhöhten Risiko für das Auftreten von Bluthochdruck assoziiert ist, so die Autoren. Laut den Experten besteht Bedarf an der Klärung der Frage, wie die Wahl der Behandlung die Risikofaktoren für kardiovaskuläre Ereignisse bei AS beeinflusst. Weitere Studien sind erforderlich, die sich auf die Präzisionsmedizin und die Vorhersage von Risiko und Nutzen für Patienten konzentrieren.

Dr. Maddalena Angela Di Lellis, Tübingen 